\title{
Recent findings about combined size and rate effects on material properties
}

\author{
Z. Chen ${ }^{1,2}$ \& S. Jiang ${ }^{1,2}$ \\ ${ }^{1}$ Department of Engineering Mechanics, \\ Dalian University of Technology, China \\ ${ }^{2}$ Department of Civil \& Environmental Engineering, \\ University of Missouri, USA
}

\begin{abstract}
The need for modeling and simulating multiscale structural responses to extreme loading conditions has brought about the challenging tasks of bridging different spatial and temporal scales within a unified framework. Based on the available experimental and computational capabilities, a simple approach has been proposed to formulate a hyper-surface in both spatial and temporal domains to predict combined specimen size and loading rate effects on the material properties. A systematic investigation has been performed over the last several years to understand the combined size, rate and thermal effects on the properties and deformation patterns of representative materials with different nanostructures and under various types of loading conditions. In this presentation, recent findings are presented about combined size and rate effects on the material properties of single crystal copper nanobeams under impact loading, with a focus on the link between the inverse Hall-Petch phenomenon and classical Hall-Petch phenomenon. It appears from the preliminary results that the inverse Hall-Petch behavior in single crystal materials is mainly due to the formation and evolution of disordered atoms as compared with the physics behind the inverse Hall-Petch behavior in nanocrystalline materials.
\end{abstract}

Keywords: size effect, rate effect, thermal effect, multiscale modeling and simulation, nanostructures. 


\section{Introduction}

As reviewed by Chen et al. [1], much research has been conducted to investigate the rate-dependence and size-dependence of material properties, respectively. However, the majority of multiscale modeling efforts are related to the scale effect in the spatial domain with the loading rate being assumed to be quasistatic. The recent need for modeling and simulating multiscale structural responses to extreme loading conditions, such as the infrastructural sensor network response to blast/impact as well as nano energetic structural responses, has brought about the challenging tasks of bridging different spatial and temporal scales within a unified framework. Although the molecular level investigations have been conducted recently to explore the size and rate effects, the length and time scales that can be probed by the molecular level simulations are still fairly limited due to the limitation of computational capability. In addition, not only the loading rate but also the specimen size used in the current molecular dynamics (MD) simulation cannot be handled by the existing experimental techniques for quasi-static, dynamic and impact tests. A specimen of finite size must be employed in the bar and plate impact experiments to investigate the ratedependent mechanical properties under the loading rate which is way below that used in the MD simulation reported so far.

Based on the available experimental and computational capabilities, a hypersurface in both spatial and temporal domains has been proposed to model combined size and rate effects on the mechanical properties of engineering materials [1]. To demonstrate the features of the proposed procedure, a hypersurface for tungsten single crystals of various sizes under various loading rates along the uniaxial tensile loading path has been formulated based on the sizedependent quasi-static strength of tungsten, as shown in Figs. 1(a) and (b).

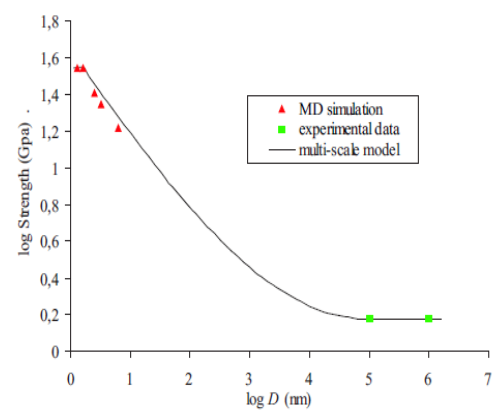

(a)

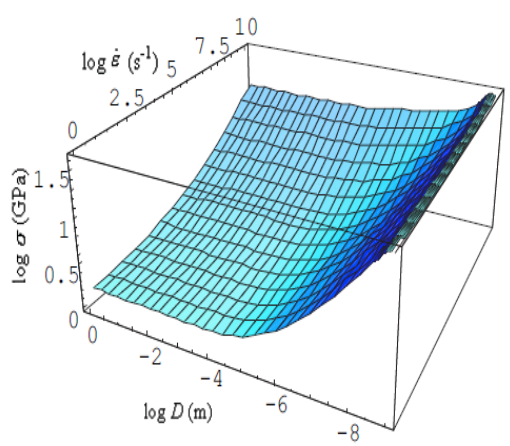

(b)

Figure 1: (a) Size-dependent quasi-static strength of tungsten (b): Hyper-surface for the strength as a function of size and strain rate [1]. 
The procedure for formulating the hyper-surface is summarized as follows:

1. Under quasi-static loading, the continuum level data are obtained by using the Weibull statistics, and the molecular level data are determined with molecular mechanics simulation tools.

2. At the continuum level, quasi-static, dynamic and impact experiments are conducted to determine the rate-dependent responses.

3. Assuming that there is no sudden change in material properties, i.e., no local minina or maxima, a monotonic hypersurface of material properties, as a function of the rate and size, could be formulated via analytical geometry for given boundary conditions.

4. For different materials, the hypersurface exhibits different changes in curvature, which must be determined by selected data points on the surface.

To model combined size, rate and thermal effects, a 4-dimensional hypersurface has also been formulated, as shown in Figs. 2(a)-(c), for tungsten single crystals subjected to uniaxial tensile loading conditions [2]. Due to the 4dimensional nature of the hyper-surface, 3-dimensional surfaces are constructed with one of the model parameters being adjusted. As can be seen from the three cases shown in Fig. 2, the size effect is dominant as compared with the rate and thermal effects.

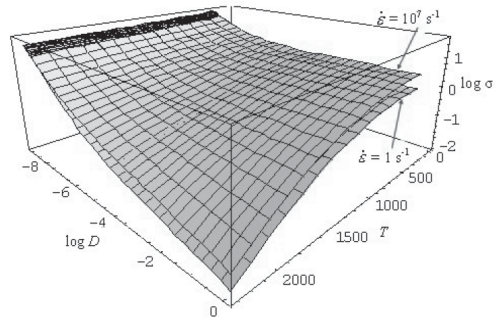

(a)

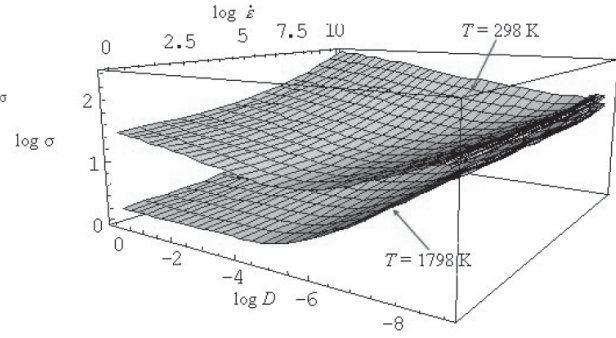

(b)

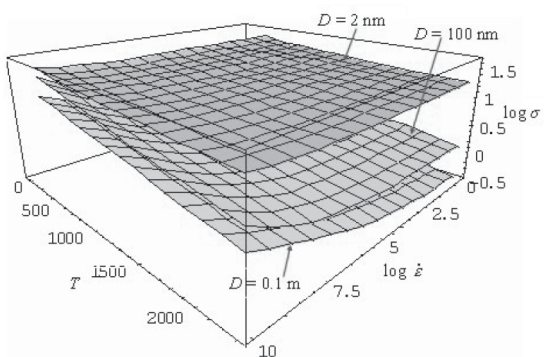

(c)

Figure 2: (a) Hyper-surface for the strength as a function of size and temperature at different rates. (b) Hyper-surface for the strength as a function of size and rate at different temperatures. (c) Hypersurface for the strength as a function of rate and temperature at different sizes [2]. 
Since the hyper-surface approach was proposed in 2005, a systematic investigation has been performed to understand the combined size, rate and thermal effects on the properties and deformation patterns of representative materials with different nanostructures and under various types of loading conditions, with or without introducing imperfections [3-16; among others]. It appears from the research results obtained so far that the proposed approach might provide an effective means to bridge different spatial and temporal scales in a unified multiscale modeling framework for different environments. Continued efforts are being made to improve the proposed approach for the applications to general cases. One of the remaining key issues to be addressed is on the link between the inverse Hall-Petch phenomenon and usual Hall-Petch phenomenon. As reviewed by Carlton and Ferreira [17], much research has been conducted for more than 10 years on the inverse Hall-Petch effect as observed for nanocrystalline materials, but controversy still persists about the operating mechanisms. Our recent work on the impact response of single crystal copper $(\mathrm{Cu})$ nanobeams [18] reveals that the link between the inverse Hall-Petch and usual Hall-Petch effects might be better explored with MD simulations of single crystals subjected to impact loadings. Hence, the remaining sections of the paper are devoted to our new findings based on the recent results in [18].

\section{Simulation approach}

We considered a $\mathrm{Cu}$ single-crystal flyer with width $\times$ height $\times$ thickness $l_{\mathrm{x}} \times l_{\mathrm{y}} \times l_{\mathrm{z}}$ impacting along the $x$-direction onto a $\mathrm{Cu}$ single-crystal target sample of size $L_{x} \times L_{y} \times L_{z}$, as illustrated in the inset of Fig. 3(a). The system is periodic along the $z$-direction but non-periodic along $x$ and $y$, and therefore corresponds to the faceto-face impact between two infinitely long nanobeams. The aspect ratio between the flyer and target is defined as the ratio $l_{y} / L_{y}$ between flyer and target heights. All simulations were performed using the LAMMPS code [19] with the embedded-atom method (EAM) force field $[20,21]$. There were three stages of the simulations. First, the lattice parameter $a$ for a 3-D periodic cubic crystal containing 2048 atoms was calculated from an isothermal-isobaric (NPT) simulation of length $2.892 \mathrm{~nm}$ for $0.5 \mathrm{~ns}$ at $T=298 \mathrm{~K}$ and zero pressure. Next, starting with the flyer and target separated by a face-to-face distance of $7.26 \mathrm{~nm}$, the pre-impact simulation cell was equilibrated for $25 \mathrm{ps}$ at $T=298 \mathrm{~K}$ in the isochoric-isothermal $(N V T)$ ensemble using the Nosé-Hoover thermostat [22, 23]. This step was performed to relieve the stresses resulting from the introduction of free surfaces in the $x$ - and $y$-directions. Finally, an initial impact velocity was added to the $x$-component of the velocities of the atoms comprising the flyer in the thermalized system after which the impact process was simulated in the isochoric-isoenergetic (NVE) ensemble for 15 ps. All simulations were performed using the velocity Verlet integrator with a time step of 0.5 fs. The widths of the flyer and target sample were chosen to be $l_{x}=10 a=3.63 \mathrm{~nm}$ and $L_{x}=40 a=14.52 \mathrm{~nm}$, respectively, for all the simulations. Common neighbor analysis (CAN) [24] was used to study the evolution of the nanostructure; in the results discussed below fcc atoms are shown as grey, hexagonal close-packed 
(hcp) atoms blue, other twelvefold-coordinated atoms green, and the remaining atoms (classified as disordered) brown. A local crystalline structure is classified as an intrinsic stacking fault if it contains two adjacent layers of atoms in the local hcp structure, an extrinsic stacking fault if it contains two hcp layers separated by a single fcc layer, and a twin boundary if it consists of a single hep layer. Disordered or nonstructured atoms are typically located at surfaces or dislocation cores $[16,25]$.

Due to the periodic boundary conditions in the $z$-direction, a suitable sample thickness $l_{z}=L_{z}$ along the length of the nanobeams has to be used to avoid the finite-size effect on the formation and growth of geometrically necessary dislocations. The effect of sample thickness on the impact stress and corresponding deformation patterns is shown in Figs. 3(a)-(c) for the aspect ratio $l_{y} / L_{y}=20 a / 40 a=1: 2$ and impact velocity $1000 \mathrm{~m} \cdot \mathrm{s}^{-1}$. The impact pressure is defined to be the negative of the principal stress along the impact direction (i.e., along the $x$-axis), calculated from the average stress tensor evaluated for a subset containing 761 atoms initially arranged in an orthorhombic $6 a \times 6 a \times 4 a$ Lagrangian subvolume, using the stress calculation method described previously $[15,16]$. The subset is initially centered on the centerline of the target at the distance $5 a$ from the impact surface and is tracked during the impact process. The size of the subset is chosen such that increasing the number of atoms in it does not lead to significant change in the calculated stress value. The time origin for trajectory analysis is taken to be the instant at which the flyer contacts the target.

From Fig. 3(a) we conclude that increasing the system thickness beyond $40 \mathrm{a}$ does not significantly affect the pressure time history. By contrast, dislocation distributions differ considerably for the systems with thicknesses $10 a$ and $40 a$ (see Figs. 3(b) and 1(c), respectively). For both thicknesses gliding dislocations form near the impact surface at short times (left-hand panels); dislocation cores nucleate at the impact surface and then glide along $\langle 111\rangle$, forming stacking faults on $\{111\}$ close-packed planes. However, the samples with different thicknesses exhibit clearly distinct deformation patterns. Whereas the stacking faults in the $10 a$-thick sample are arranged in an essentially herringbone pattern (see Fig. 3(b), center panel), for the $40 a$-thick sample the dislocation pattern is much less ordered with significant crisscrossing (see Fig. 3(c), center panel). These differences are due to the formation and evolution of geometrically necessary dislocations in the thinner sample (due to the smaller periodic length $L_{z}$ ) that results in a regular arrangement of adjacent dislocation zones.

Figures 3(d) and 1(e) further illustrate that the stress state in the thinner sample (see Fig. 3(d)) is more liquid-like than in the thicker sample (see Fig. 3(e)) insofar as the difference between the maximum and minimum principal stresses near the impact surface decreases with the decrease of the sample thickness. Figure 3(f) demonstrates that the subset of atoms used to calculate the stress near the impact surface remains intact and that there is no localized deformation in the atomic configuration of the subset within 2 ps of impact. As a result, the subset chosen for evaluating the average stress tensor should yield a meaningful stress measure within the first 2 ps (the time interval of interest for 
the peak stress results to be discussed later) after impact occurs. As shown in the right-hand panels of Figs. 3(b) and 3(c), substantial recovery of the fcc crystal structure occurs when the release wave moves through the target sample. The time histories for impact stress, number fractions of hep atoms (not shown), and number fractions of disordered atoms (not shown) are nearly the same for simulation cell thicknesses $40 a$ and $60 a$. Therefore, the flyer and sample thickness $l_{z}=L_{z}=40 a$ is chosen for all subsequent calculations.

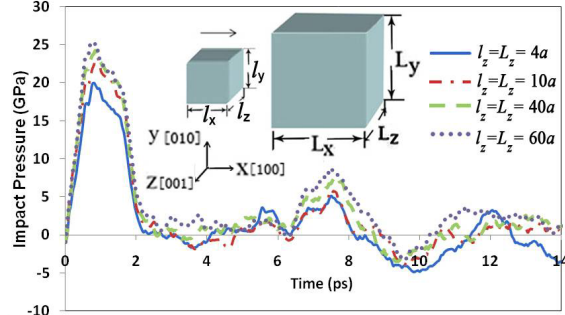

(a)

(b)

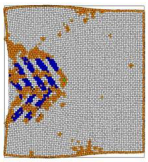

(c)

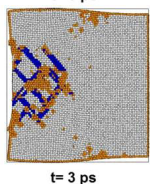

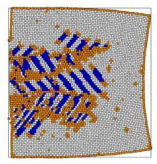

$t=6 p s$

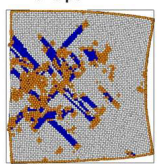

$\mathrm{t}=6 \mathrm{ps}$

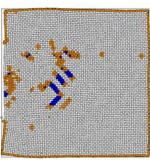

$t=12$ ps

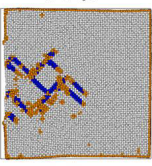

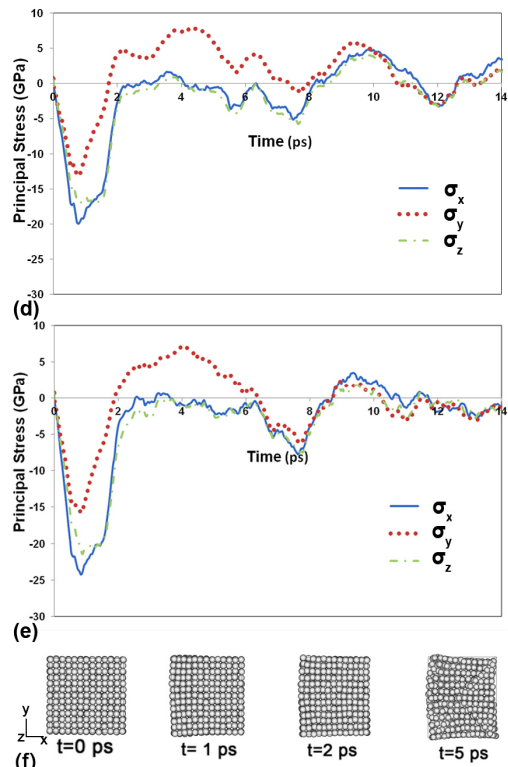

Figure 3: (a): The effect of sample thickness on the impact pressure for the aspect ratio 1:2 and impact velocity $1000 \mathrm{~m} \cdot \mathrm{s}^{-1}$, with the inset being a depiction of the simulation model; (b) and (c): Target deformation patterns for the sample thicknesses of (b) $L_{z}=1.452 \mathrm{~nm}$ and (c) $L_{z}=$ $14.52 \mathrm{~nm}$ at the post-impact times 3,6 and $12 \mathrm{ps}$, respectively. (d) and (e): Time histories of the principal stresses in the target for sample thicknesses (d) $L_{z}=1.452 \mathrm{~nm}$ and (e) $L_{z}=14.52 \mathrm{~nm}$; (f): Instantaneous atomic configurations, at times $t=0,1,2$, and $5 \mathrm{ps}$, of the Lagrangian subset of atoms chosen for evaluating the average stress tensor [18].

It should be pointed out that the continuum equation $|\sigma|=\left|\rho c_{L} \dot{u}\right|$ that relates the longitudinal stress $\sigma$ to the particle velocity $\dot{u}$ through the mechanical impedance $\rho c_{L}$, where $c_{L}$ is the longitudinal wave speed, is based on the halfspace derivation [26, 27]. Clearly, this equation is not applicable to the finite nanobeam structures considered here because of the finite space. As can be seen from Fig. 3(a), although the peak pressure differs with sample thickness, the time at which the peak stress occurs is independent of sample thickness; hence, the 
shock wave speed in the simulations is independent of sample thickness. By contrast, for a given particle velocity, which in the present case is simply onehalf of the impact velocity because the flyer and target materials are the same, the wave speed predicted by the equation at the beginning of the paragraph will be different for different sample thicknesses due to the dependence of the peak stress on the sample thickness in the simulations. Hence, the continuum equations governing the impact responses of semi-infinite systems cannot be directly applied to the finite nanoscale systems of interest.

\section{Findings and discussion}

As shown in Fig. 3(a), the impact stress decreases with the decease of sample thickness. To explore the link between the inverse Hall-Petch and classical HallPetch effects, selected MD simulations are performed as specified in Table 1.

To understand both the flyer size $\left(l_{y}\right)$ and loading rate effects on the impact stress, selected simulations are divided into 4 groups (1-5, 6-10, 11-15 and 1520) for respective impact velocities of $500 \mathrm{~m} / \mathrm{s}, 1000 \mathrm{~m} / \mathrm{s}, 2000 \mathrm{~m} / \mathrm{s}$ and $4000 \mathrm{~m} / \mathrm{s}$. Figure 4 demonstrates both the size and rate effects on the peak impact stress in the target. Due to the large difference between the impact stresses for impact velocities of $500 \mathrm{~m} / \mathrm{s}$ and $4000 \mathrm{~m} / \mathrm{s}$, small and large scales for the stress are used in Figs. 4(a) and (b), respectively, for the purpose of clarification. As can be seen, there is an obvious transition from the inverse Hall-Petch to the usual HallPetch behavior around $l_{y}=40 a$ if the impact velocity is less than $2000 \mathrm{~m} / \mathrm{s}$. The increase of loading rates results in less deviation from the classical Hall-Petch relation, which is consistent with what has been observed for nanocrystalline materials [17]. However, the physics behind the transition for nanocrystalline materials might not be the same as that for single crystal materials considered here because any argument based on the grain boundary will not hold any more. Since the response to the impact velocity larger than $2000 \mathrm{~m} / \mathrm{s}$ appears to deviate from the transition at $l_{y}=40 a$, Figure 5 shows the target deformation patterns with time for the impact velocity of $2000 \mathrm{~m} / \mathrm{s}$. As can be observed from Fig. 5(b), there is a change from fcc to bcc (green) structures surrounded by disordered atoms when the peak pressure is reached. The impact pressure is then decreased with the evolution of dislocation. Hence, the interaction between ordered and disordered atoms might play an important role in the load-carrying capacity. The question is how the peak pressure in the target is affected by the flyer size, and how the transition from the inverse Hall-Petch to the classical Hall-Petch phenomenon would occur.

Figure 6 illustrates the flyer size effect on the target deformation patterns when the peak impact pressure is reached for the impact velocity of $2000 \mathrm{~m} / \mathrm{s}$. As can be observed from Fig. 6, the density of disordered atoms in the region where the impact pressure is measured decreases with the increase of the flyer size (quantitative calculation, not shown here, confirms this observation).

The time history of the impact pressure in the target for the flyer size of $l_{y}=40 a$ is shown in Fig. 7 for different impact velocities, with the corresponding target deformation patterns given in Fig. 8. Although the rate-dependence for 
46 High Performance Structure and Materials VI

Table 1: $\quad$ Specification of selected MD simulations, with $a=0.363 \mathrm{~nm}$.

\begin{tabular}{|c|c|c|c|c|}
\hline $\begin{array}{c}\text { Simulation } \\
\text { No. }\end{array}$ & $\begin{array}{c}\text { Flyer size } \\
l_{\mathrm{x}} \times l_{\mathrm{y}} \times l_{\mathrm{z}}\end{array}$ & $\begin{array}{c}\text { Target size } \\
L_{\mathrm{x}} \times L_{\mathrm{y}} \times L_{\mathrm{z}} \\
\end{array}$ & $\begin{array}{c}\text { Impact velocity } \\
(\mathrm{m} / \mathrm{s})\end{array}$ & $\begin{array}{c}\text { Aspect ratio } \\
\left(l_{\mathrm{y}} / L_{\mathrm{y}}\right) \\
\end{array}$ \\
\hline 1 & $\begin{array}{c}10 a \times 10 \\
a \times 10 a\end{array}$ & $\begin{array}{c}40 a \times 40 \\
a \times 80 a\end{array}$ & 500 & $1: 8$ \\
\hline 2 & $\begin{array}{c}10 a \times 10 \\
a \times 20 a\end{array}$ & $\begin{array}{c}40 a \times 40 \\
a \times 80 a\end{array}$ & 500 & $1: 4$ \\
\hline 3 & $\begin{array}{c}10 a \times 10 \\
a \times 40 a\end{array}$ & $\begin{array}{c}40 a \times 40 \\
a \times 80 a\end{array}$ & 500 & $1: 2$ \\
\hline 4 & $\begin{array}{c}10 a \times 10 \\
a \times 60 a\end{array}$ & $\begin{array}{c}40 a \times 40 \\
a \times 80 a\end{array}$ & 500 & $3: 4$ \\
\hline 5 & $\begin{array}{c}10 a \times 10 \\
a \times 80 a\end{array}$ & $\begin{array}{c}40 a \times 40 \\
a \times 80 a\end{array}$ & 500 & $1: 1$ \\
\hline 6 & $\begin{array}{c}10 a \times 10 \\
a \times 10 a\end{array}$ & $\begin{array}{c}40 a \times 40 \\
a \times 80 a\end{array}$ & 1000 & $1: 8$ \\
\hline 7 & $\begin{array}{c}10 a \times 10 \\
a \times 20 a\end{array}$ & $\begin{array}{c}40 a \times 40 \\
a \times 80 a\end{array}$ & 1000 & $1: 4$ \\
\hline 8 & $\begin{array}{c}10 a \times 10 \\
a \times 40 a\end{array}$ & $\begin{array}{c}40 a \times 40 \\
a \times 80 a\end{array}$ & 1000 & $1: 2$ \\
\hline 9 & $\begin{array}{c}10 a \times 10 \\
a \times 60 a\end{array}$ & $\begin{array}{c}40 a \times 40 \\
a \times 80 a\end{array}$ & 1000 & $3: 4$ \\
\hline 10 & $\begin{array}{c}10 a \times 10 \\
a \times 80 a\end{array}$ & $\begin{array}{c}40 a \times 40 \\
a \times 80 a\end{array}$ & 1000 & $1: 1$ \\
\hline 11 & $\begin{array}{c}10 a \times 10 \\
a \times 10 a\end{array}$ & $\begin{array}{c}40 a \times 40 \\
a \times 80 a\end{array}$ & 2000 & $1: 8$ \\
\hline 12 & $\begin{array}{c}10 a \times 10 \\
a \times 20 a\end{array}$ & $\begin{array}{c}40 a \times 40 \\
a \times 80 a\end{array}$ & 2000 & $1: 4$ \\
\hline 13 & $\begin{array}{c}10 a \times 10 \\
a \times 40 a\end{array}$ & $\begin{array}{c}40 a \times 40 \\
a \times 80 a\end{array}$ & 2000 & $1: 2$ \\
\hline 14 & $\begin{array}{c}10 a \times 10 \\
a \times 60 a\end{array}$ & $\begin{array}{c}40 a \times 40 \\
a \times 80 a\end{array}$ & 2000 & $3: 4$ \\
\hline 15 & $\begin{array}{c}10 a \times 10 \\
a \times 80 a\end{array}$ & $\begin{array}{c}40 a \times 40 \\
a \times 80 a\end{array}$ & 2000 & $1: 1$ \\
\hline 16 & $\begin{array}{c}10 a \times 10 \\
a \times 10 a\end{array}$ & $\begin{array}{c}40 a \times 40 \\
a \times 80 a\end{array}$ & 4000 & $1: 8$ \\
\hline 17 & $\begin{array}{c}10 a \times 10 \\
a \times 20 a\end{array}$ & $\begin{array}{c}40 a \times 40 \\
a \times 80 a\end{array}$ & 4000 & $1: 4$ \\
\hline 18 & $\begin{array}{c}10 a \times 10 \\
a \times 40 a\end{array}$ & $\begin{array}{c}40 a \times 40 \\
a \times 80 a\end{array}$ & 4000 & $1: 2$ \\
\hline 19 & $\begin{array}{c}10 a \times 10 \\
a \times 60 a\end{array}$ & $\begin{array}{c}40 a \times 40 \\
a \times 80 a\end{array}$ & 4000 & $3: 4$ \\
\hline 20 & $\begin{array}{c}10 a \times 10 \\
a \times 80 a\end{array}$ & $\begin{array}{c}40 a \times 40 \\
a \times 80 a\end{array}$ & 4000 & $1: 1$ \\
\hline
\end{tabular}



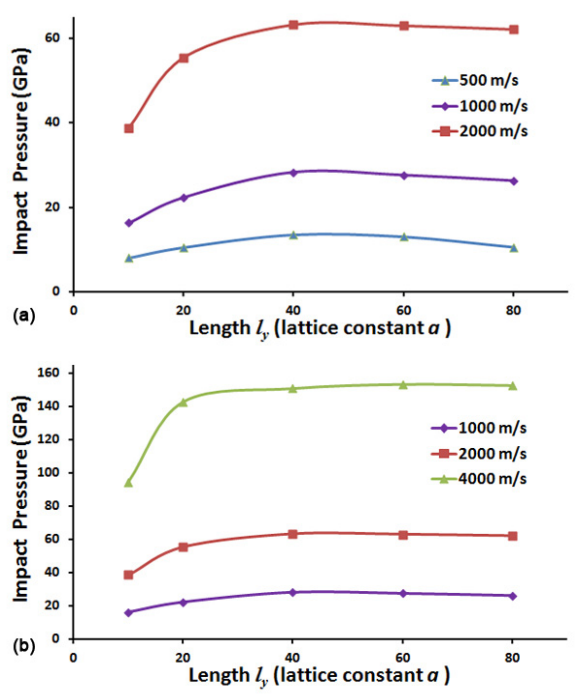

Figure 4: The maximum impact pressure reached in the target of different sizes, for different impact velocities of (a) $500 \mathrm{~m} / \mathrm{s}, 1000 \mathrm{~m} / \mathrm{s}$ and $2000 \mathrm{~m} / \mathrm{s}$, and (b) $1000 \mathrm{~m} / \mathrm{s}, 2000 \mathrm{~m} / \mathrm{s}$ and $4000 \mathrm{~m} / \mathrm{s}$, respectively.

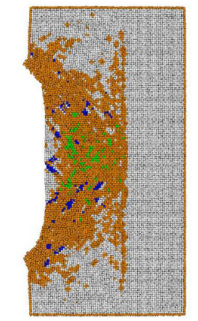

(c)

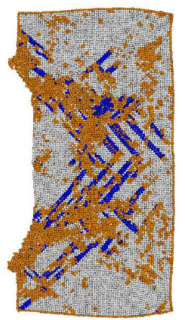

(d)

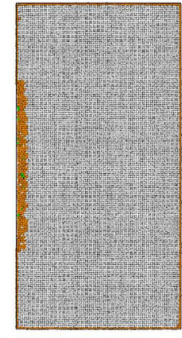

(a)

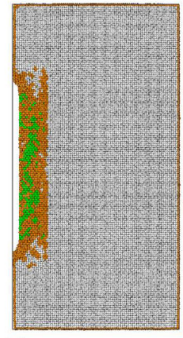

(b)

Figure 5: Target deformation patterns for $1_{\mathrm{y}}=40 a$ and impact velocity of $2000 \mathrm{~m} / \mathrm{s}$, with (a) $\mathrm{t}=\sim 0.05 \mathrm{ps}$ (before the peak impact pressure), (b) $\mathrm{t}=\sim 0.45 \mathrm{ps}$ (around the peak impact pressure), (c) $\mathrm{t}=\sim 1.65 \mathrm{ps}$ and (d) $t=\sim 3.65$ ps (after the peak impact pressure).

single crystal materials is the same as that for nanocrystalline materials, the physics behind the size-dependence appears to be different due to the absence of grain boundary in single crystal materials.

As shown in Fig. 6, the reason why the density of disordered atoms in the region where the impact pressure is calculated for $l_{y}=20 a$ is highest is because of its closest distance between the flyer corners. With the increase of the flyer size, the density of disordered atoms becomes small so that the peak impact stress is increased until the large flyer size facilitates the formation and evolution of 
dislocation. Figure 8 further demonstrates that the transition from the inverse Hall-Petch to classical Hall-Petch behavior might be due to the interaction between the formation of highly disordered atoms in a localized region and the space available for the formation and evolution of dislocation, which is ratedependent.

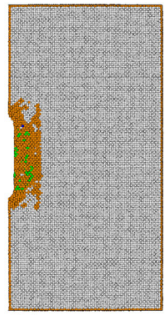

(a)

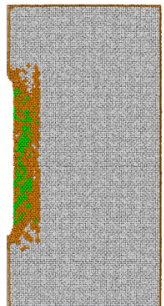

(b)

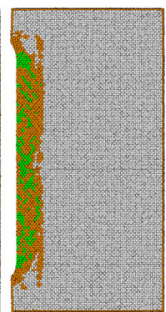

(c)

Figure 6: Target deformation patterns around the peak impact pressure with the impact velocity of $2000 \mathrm{~m} / \mathrm{s}$ for the cases of (a) $1_{\mathrm{y}}=20 a$ (b) $1_{\mathrm{y}}=$ $40 a$ and (c) $1_{\mathrm{y}}=60 a$, respectively.
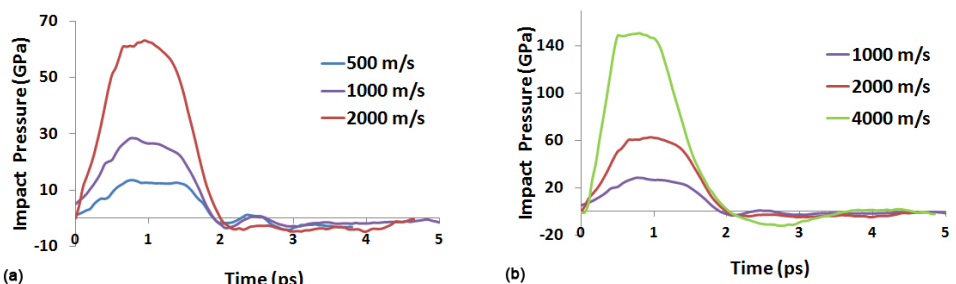

Figure 7: Time history of the impact pressure for the cases of $1_{\mathrm{y}}=40 \mathrm{a}$ with different impact velocities of (a) $500 \mathrm{~m} / \mathrm{s}, 1000 \mathrm{~m} / \mathrm{s}$ and $2000 \mathrm{~m} / \mathrm{s}$ and (b) $1000 \mathrm{~m} / \mathrm{s}, 2000 \mathrm{~m} / \mathrm{s}$ and $4000 \mathrm{~m} / \mathrm{s}$, respectively.

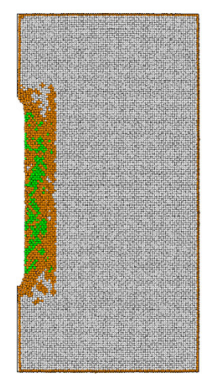

(c)

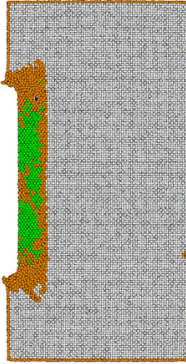

(d)

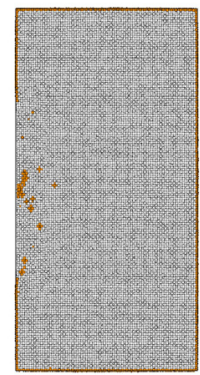

(a)

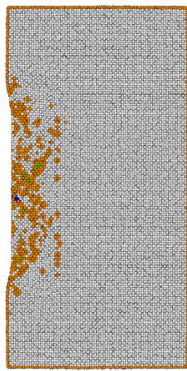

(b)

Figure 8: Target deformation patterns around the peak impact pressure for $\mathrm{l}_{\mathrm{y}}=$ $40 a$ with different impact velocities of (a) $500 \mathrm{~m} / \mathrm{s}$, (b) $1000 \mathrm{~m} / \mathrm{s}$, (c) $2000 \mathrm{~m} / \mathrm{s}$ and (d) $4000 \mathrm{~m} / \mathrm{s}$, respectively. 


\section{Conclusion and future tasks}

Recent findings have been described about combined size and rate effects on the impact response between single crystal $\mathrm{Cu}$ flyer and target. An attempt has been made to explore the physics behind the link between the inverse Hall-Petch phenomenon and classical Hall-Petch phenomenon. It appears from the preliminary results that the inverse Hall-Petch behavior in single crystal materials is mainly due to the formation and evolution of disordered atoms as compared with the physics behind the inverse Hall-Petch behavior in nanocrystalline materials. Based on the findings, a hyper-surface model is being developed and will be presented in the conference.

\section{References}

[1] Chen, Z., Shen, L., Gan, Y. and Fang, H.E., Hyper-Surface for the Combined Loading Rate and Specimen Size Effects on the Material Properties. International Journal for Multiscale Computational Engineering, 3(4), pp. 451-461, 2005.

[2] Chen, Z., Shen, L., Dai, H. and Gan, Y., Recent Efforts in Modeling Combined Rate, Size and Thermal Effects on Single Crystal Strength. Review on Advanced Materials Science, 13, pp. 27-32, 2006.

[3] Shen, L. and Chen, Z., A Numerical Study of the Size and Rate Effects on the Mechanical Response of Single Crystal Diamond and UNCD Films. International Journal of Damage Mechanics, 15(2), pp. 169-195, 2006.

[4] Shen, L. and Chen, Z., Study of the Combined Temperature, Rate and Size Effects on the Tungsten Crystalline Block Strength. Proceedings of the Institution of Mechanical Engineers, Part N, Journal of Nanoengineering and Nanosystems, 219(3), pp. 111-122, 2006.

[5] Guo, Y., Zhuang, Z., Li, X.Y. and Chen, Z., An Investigation of the Combined Size and Rate Effects on the Mechanical Responses of FCC Metals. International Journal of Solids and Structures, 44(3-4), pp. 11801195, 2007.

[6] Shen, L. and Chen, Z., A Numerical Study of Combined Rate, Size and Thermal Effects on the Responses of Ultrananocrystalline Diamond. Key Engineering Materials, 334-335, pp. 621-624, 2007.

[7] Shen, L. and Chen, Z., An Investigation of Grain Size and Nitrogen-Doping Effects on the Mechanical Properties of Ultrananocrystalline Diamond Films. International Journal of Solids and Structures, 44(10), pp. 33793392, 2007.

[8] Shen, L. and Chen, Z., A Study of the Loading Path and Crystal Orientation Effects on Size-Dependent Limit Strength. Engineering Fracture Mechanics, 74(7), pp. 1190-1202, 2007.

[9] Shen, L. and Chen, Z., The Loading History and Crystal Orientation Effects on the Size-Dependency of Single Crystal Diamond Properties. Computational Mechanics, 42(4), pp. 619-629, 2008. 
[10] Chen, Z., Gan, Y. and Labuz, J.F., Analytical and Numerical Study of the Size Effect on the Failure Response of Hierarchical Structures. International Journal for Multiscale Computational Engineering, 6(4), pp. 339-348, 2008.

[11] Shen, L. and Chen, Z., A Study of Mechanical Properties of Pure and Nitrogen-Doped Ultrananocrystallin Diamond Films under Various Loading Conditions. International Journal of Solids and Structures, 46(34), pp. 811-823, 2009.

[12] Shen, L. and Chen, Z., An Investigation of Combined Size, Rate and Thermal Effects on the Material Properties of Single Crystal Diamond. International Journal of Materials and Product Technology, 34(1-2), pp. 111-130, 2009.

[13] Shen, L. and Chen, Z., A Numerical Study of the Imperfection Effect on Ultrananocrystalline Diamond Properties under Different Loading Paths and Temperatures. Composites Science and Technology, 69(13), pp. 20752080, 2009.

[14] Shen, L. and Chen, Z., Loading History Effect on Size-Dependent Shear Strength of Pure and Nitrogen-Doped Ultrananocrystalline Diamond. Mechanics of Advanced Materials and Structures, 16(7), pp. 504-515, 2009.

[15] Shen, L.M. and Chen, Z., Molecular Dynamics Study of the Specimen Size and Imperfection Effects on the Failure Responses of Multi-Nanobar Structures. International Journal for Multiscale Computational Engineering, 8(2), pp. 181-194, 2010.

[16] Jiang, S., Zhang, H.W., Zheng, Y.G. and Chen, Z., Loading Path Effect on the Mechanical Behavior and Fivefold Twinning of Copper Nanowires. Journal of Physics D: Applied Physics, 43(33), 335402 (9pp), 2010.

[17] Carlton, C.E., and Ferreira, P.J., What is behind the inverse Hall-Petch effect in nanocrystalline materials? Acta Materialla, 55(11), pp. 3749-3756, 2007

[18] Chen, Z., Jiang, S., Gan, Y., Oloriegbe, S., Sewell, T. and Thompson, D., Size Effects on the Impact Response of Copper Nanobeams. To appear in Journal of Applied Physics, 2012.

[19] Plimpton, S. J. Fast parallel algorithms for short-range molecular dynamics, Journal of Computational Physics, 117(1), pp. 1-19, http://lammps.sandia.gov, 1995.

[20] Daw, M. S. and Baskes, M. I., Embedded-atom method: derivation and application to impurities, surfaces, and other defects in metals. Physical Review B, 29(12), pp. 6443-6453, 1984.

[21] Foiles, S. M., Baskes, M. I. and Daw, M. S., Embedded-atom-method functions for the fcc metals $\mathrm{Cu}, \mathrm{Ag}, \mathrm{Au}, \mathrm{Ni}, \mathrm{Pd}, \mathrm{Pt}$, and their alloys. Physical Review B, 33(12), pp. 7983-7991, 1986.

[22] Nosé, S., A unified formulation of the constant temperature molecular dynamics methods. Journal of Chemical Physics., 81(1), pp. 511-519, 1984. 
[23] Hoover, W. G., Canonical dynamics: Equilibrium phase-space distributions. Physical Review A, 31(3), pp. 1695-1697, 1985.

[24] Tsuzuki, H., Branicia, P. S. and Rino, J. P., Structural characterization of deformed crystals by analysis of common atomic neighborhood. Computer Physics Communications, 177(6), pp. 518-523, 2007.

[25] Jiang, S., Zhang, H., Zheng, Y. and Chen, Z., Atomistic study of the mechanical response of copper nanowires under torsion. Journal of Physics D: Applied Physics., 42(13), 135408(6pp), 2009.

[26] Achenbach, J.D., Wave Propagation in Elastic Solids, Elsevier Science Publishers B.V., 1984.

[27] Zukas, J. A., Nicholas, T., Swift, H. F., Greszczuk, L.B. and Curran, D. R., Impact Dynamics, Krieger Publishing Company, 1992. 